Pacific Journal of Mathematics

FIXED POINTS OF AUTOMORPHISMS OF COMPACT LIE

GR OPS 


\title{
FIXED POINTS OF AUTOMORPHISMS OF COMPACT LIE GROUPS
}

\author{
ROBERT F. BROWN
}

\begin{abstract}
Hopf's proof that the real Cech cohomology $H^{*}(G)$ of a compact, connected Lie group $G$ is an exterior algebra with odd-dimensional generators was followed by a demonstration that the number of such generators is equal to the rank of the group, that is, to the dimension of a maximal torus. We show that the latter result is a special case of a relationship between an automorphism of such a group and the automorphism it induces on the cohomology.
\end{abstract}

1. Introduction. For a set $X$ and a function $f: X \rightarrow X$, let $\Phi(f)$ denote the set of fixed points of $f$ : those $x \in X$ for which $f(x)=x$. If $X$ is a topological group and $f$ is a homomorphism, we use the symbol $\Phi_{0}(f)$ for the component of the group $\Phi(f)$ which contains the identity element of $X$. By a graded vector space $V$ we mean a sequence $\left\{V_{0}, V_{1}, V_{2}, \cdots\right\}$ of (real) vector spaces. The dimension of $V$ is the sum of the dimensions of the $V_{i}$. A subspace of $V$ is a graded vector space $V^{\prime}=\left\{V_{\imath}^{\prime}\right\}$ such that $V_{i}^{\prime}$ is a subspace of $V_{\imath}$; for all $i$.

Now let $G$ be a compact, connected Lie group and let $h$ be an automorphism of $G$. Denote by $P H^{*}(G)$ the graded vector subspace of primitives ${ }^{1}$ in the Hopf algebra $H^{*}(G)$ and let $P h^{*}$ be the restriction to $P H^{*}(G)$ of the automorphism $h^{*}: H^{*}(G) \rightarrow H^{*}(G)$ induced by $h$.

The main result of this paper is

THEOREM 1.1. Let $G$ be a compact connected Lie group and let $h$ be an automorphism of $G$. Then the rank of the Lie group $\Phi_{0}(h)$ is equal to the dimension of the graded vector space $\Phi\left(P h^{*}\right)$.

If $h$ is the identity automorphism, then $\Phi(h)=G$ while $\Phi\left(P h^{*}\right)=$ $P H^{*}(G)$. Since $H^{*}(G)$ may be generated by primitives, the number of generators is the dimension of $P H^{*}(G)$. Thus Hopf's result in [4] on the rank of a compact Lie group is this case of Theorem 1.1.

The next section presents a digression concerning the kernel of an endomorphism of a compact, connected abelian topological group. The setting is more general than is necessary for the later sections because some readers may find this material of independent interest. The proof of Theorem 1.1 is accomplished in $\S 3$. The remaining

1 A primitive in $H^{*}(G)$ is an element $z$ for which $m^{*}(z)=1 \otimes z+z \otimes 1$; where $m^{*}: H^{*}(G) \rightarrow H^{*}(G) \otimes H^{*}(G)$ is induced by the multiplication $m: G \times G \rightarrow G$ of the group. 
sections discuss consequences of the main result. Section 4 demonstrates that the existence of an automorphism $h$ on a compact, connected Lie group $G$ such that the fixed point group of $h$ is of low rank implies that $G$ has a very restricted type of infinitesimal structure. In $\S 5$, we obtain necessary and sufficient conditions for the power map $p_{k}(x)=x^{k}$ on a Lie group with compact components to map a component onto another. This theorem extends the main result of [2] which established the conditions only for compact Lie groups. The results contained in this paper were announced in $[1]^{2}$.

2. Endomorphisms of abelian topological groups. The kernel of a homomorphism $h$ will be denoted by $\operatorname{Ker}(h)$. For a homomorphism $h$ on a topological group, the symbol $\operatorname{Ker}_{0}(h)$ will represent the component of the kernel of $h$ that contains the identity element of the group.

Let $G$ be a compact, connected abelian topological group and let $h$ be an endomorphism of $G$. Denote the character group of $G$ by $G^{\wedge}$ and write the endomorphism of $G^{\wedge}$ induced by $h$ as $h^{\wedge}$.

For a subgroup $H$ of $G$, let $i: H \rightarrow G$ be inclusion and let $\operatorname{Ann}(H)$ denote the subgroup of $G^{\wedge}$ consisting of all elements which vanish on $H$. By [6, p. 253], there is an exact sequence

$$
0 \longrightarrow \operatorname{Ann}(H) \longrightarrow G^{\wedge} \stackrel{i^{\wedge}}{\longrightarrow} H^{\wedge} \longrightarrow 0 \text {. }
$$

In particular, letting $\operatorname{Im}(h)$ be the image of $h$, we have the exact sequence

$$
0 \longrightarrow \operatorname{Ann}(\operatorname{Im}(h)) \longrightarrow G^{\wedge} \stackrel{i^{\wedge}}{\longrightarrow} \operatorname{Im}(h)^{\wedge} \longrightarrow 0 .
$$

Since $\operatorname{Im}(h)^{\wedge}$ is free, there exists a homomorphism $e: \operatorname{Im}(h)^{\wedge} \rightarrow G^{\wedge}$ such that $i^{\wedge} e$ is the identity function.

LEMMA 2.1. Let $\bar{h}: G / \operatorname{Ker}(h) \rightarrow G$ be the homomorphism induced by $h$, then $\bar{h}^{\wedge}$ takes $G^{\wedge}$ onto $(G / \operatorname{Ker}(h))^{\wedge}$.

Proof. Let $\beta: G / \operatorname{Ker}(h) \rightarrow S^{1}$ (the circle) be any element of $(G / \operatorname{Ker}(h))^{\wedge}$. Consider the diagram.

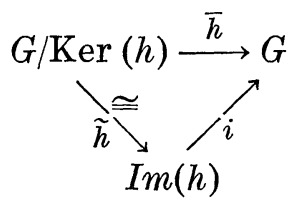

which defines $\tilde{h}$. Now define $\alpha^{\prime}=\beta \tilde{h}^{-1}: \operatorname{Im}(h) \rightarrow S^{1}$ and set $\alpha=$

${ }^{2}$ In [1] and [2], $P H^{*}(G)$ and $P h^{*}$ are denoted by $H^{*}(G)$ and $h^{*}$, respectively. 
$e\left(\alpha^{\prime}\right) \in G^{\wedge}$, then $\bar{h}^{\wedge}(\alpha)=\beta$.

Proposition 2.2. Let $G$ be a compact, connected abelian topological group, and let $h$ be an endomorphism of $G$. Then the dimension of the topological group $\mathrm{Ker}_{0}(h)$ is equal to the rank of the abelian group $\operatorname{Ker}\left(h^{\wedge}\right)$.

Proof. By the lemma, we have an exact sequence

$$
0 \longrightarrow \operatorname{Ker}\left(h^{\wedge}\right) \longrightarrow G^{\wedge} \stackrel{\bar{h}^{\wedge}}{\longrightarrow}(G / \operatorname{Ker}(h))^{\wedge} \longrightarrow 0 .
$$

Since $G$ is connected, $(G / \operatorname{Ker}(h))^{\wedge}$ is free so

$$
G^{\wedge} \cong \operatorname{Ker}\left(h^{\wedge}\right) \oplus(G / \operatorname{Ker}(h))^{\wedge} .
$$

The sequence

$$
0 \longrightarrow \operatorname{Ann}\left(\operatorname{Ker}_{o}(h)\right) \longrightarrow G^{\wedge} \longrightarrow\left(\operatorname{Ker}_{o}(h)\right)^{\wedge} \longrightarrow 0
$$

also splits, that is,

$$
G^{\wedge} \cong\left(\operatorname{Ker}_{o}(h)\right)^{\wedge} \oplus \operatorname{Ann}\left(\operatorname{Ker}_{o}(h)\right) .
$$

By [6, p. 243], Ann $\left(\operatorname{Ker}_{\circ}(h)\right)$ is the character group of $G / \operatorname{Ker}_{0}(h)$. Since $\operatorname{Ker}(h) / \operatorname{Ker}_{0}(h)$ is finite, $G / \operatorname{Ker}(h)$ and $G / \operatorname{Ker}_{o}(h)$ have the same dimension, so the abelian groups $(G / \operatorname{Ker}(h))^{\wedge}$ and $\operatorname{Ann}\left(\operatorname{Ker}_{0}(h)\right)$ have the same rank [6, p. 34 and p. 259]. We conclude that $\left(\operatorname{Ker}_{o}(h)\right)^{\wedge}$ and $\operatorname{Ker}\left(h^{\wedge}\right)$ have the same rank and the result is proved.

Let $h^{*, 1}$ denote the restriction of $h^{*}$ to $H^{1}(G)$.

Proposition 2.3. Let $G$ be a compact, connected abelian topological group, and let $h$ be an endomorphism of $G$. Then the rank of $\operatorname{Ker}\left(h^{\wedge}\right)$ is equal to the dimension of the vector space $\operatorname{Ker}\left(h^{*, 1}\right)$.

Proof. There is a natural isomorphism from $G^{\wedge}$ to $H^{1}(G ; J)$ (integer Čech cohomology) [9; Appendix 1], so we may identify $h^{\wedge}$ with the endomorphism $h_{J}^{*, 1}$ of $H^{1}(G ; J)$ induced by $h$. The Universal Coefficient Theorem implies the existence of a natural isomorphism between $H^{1}(G ; J) \otimes R\left(R=\right.$ the reals) and $H^{1}(G)$ so that $h_{J}^{*, 1} \otimes$ (identity) corresponds to $h^{*, 1}$. Consequently, $\operatorname{Ker}\left(h^{\wedge}\right)$ and $\operatorname{Ker}\left(h_{J}^{*, 1}\right)$ are isomorphic free groups and their rank is equal to the dimension of the vector space $\operatorname{Ker}\left(h^{*, 1}\right)$.

3. Proof of the main theorem. We will use the symbol Aut $(G)$ to denote the group of automorphisms of a Lie group $G$ and $\operatorname{Inn}(G)$ for the inner automorphisms. 
LEMMA 3.1. Let $G$ be a compact, connected Lie group and suppose $h \in \operatorname{Aut}(G)$ has the property that $h^{m} \in \operatorname{Inn}(G)$ for some $m \geqq 1$. Then the rank of the Lie group $\Phi_{o}(h)$ is equal to the dimension of the graded vector space $\Phi\left(P h^{*}\right)$.

Proof. By [8, p. 46], there is a subgroup $U$ of Aut $(G)$ which intersects each coset of $\operatorname{Aut}(G) / \operatorname{Inn}(G)$ in a single automorphism. Thus there exists $C_{a}$, defined by $C_{a}(x)=a x a^{-1}$ for all $x \in G$, such that $h C_{a} \in U$. Let $J_{m}$ denote the cyclic group of order $m$, then since $h^{m} \in$ Inn $(G)$ implies that $\left(h C_{a}\right)^{m}$ is the identity, we can define $\chi_{:} J_{m} \rightarrow$ Aut $(G)$ by $\chi(q)=\left(h C_{a}\right)^{q}$. Let $C_{r}^{\chi}$ denote conjugation by $r$ in $\left(G \times J_{m}\right)_{\chi}$, the semi-direct product of $G$ and $J_{m}$ induced by $\chi$. For $b=h\left(a^{-1}\right)$, we compute that $C_{b b, 1)}^{x}(x, 0)=(h(x), 0)$, so $\Phi_{0}(h)$ is isomorphic to the identity component of the centralizer of $(b, 1)$ in $\left(G \times J_{m}\right)_{\chi}$. By [7; 1.2] and [2;4.3], the rank of $\Phi_{0}(h)$ is equal to the multiplicity of +1 as an eigenvalue of $P h^{*}$. Finally, $\left(P h^{*}\right)^{m}$ is the identity transformation $E$, so that multiplicity is equal to the dimension of the kernel of $P h^{*}-E$, which is $\Phi\left(P h^{*}\right)$.

Now we turn to the proof of Theorem 1.1, that is, we obtain the conclusion of Lemma 3.1 without the hypothesis " $h^{m} \in \operatorname{Inn}(G)$ ". Let $Z$ be the identity component of the center of $G$ and let $S$ be the maximal connected semisimple normal subgroup of $G$, then $G=$ $Z S$ and $Z \cap S$ is finite. Since $Z$ and $S$ are characteristic subgroups of $G$, an automorphism $h$ of $G$ restricts to automorphisms $h_{z}$ and $h_{S}$ of $Z$ and $S$ respectively. Then

$$
\operatorname{rank}\left(\Phi_{o}(h)\right)=\operatorname{rank}\left(\Phi_{o}\left(h_{Z}\right)\right)+\operatorname{rank}\left(\Phi_{o}\left(h_{S}\right)\right)
$$

because the equation is true of the corresponding Lie algebras. By DeRham's theorem and the Künneth theorem, there are natural isomorphisms of algebras

$$
H^{*}(G) \cong H^{*}(\mathbb{S}) \cong H^{*}(Z \times S) \cong H^{*}(Z) \otimes H^{*}(S)
$$

where $\$ 8$ denotes the Lie algebra of $G$. The isomorphisms permit us to identify $P H^{*}(G)$ with $P H^{*}(Z) \oplus P H^{*}(S)$ (direct sum). Furthermore, naturality permits us to identify $P h^{*}$ with $P h_{Z}^{*} \oplus P h_{S}^{*}$, so it must be that

$$
\operatorname{dim}\left(\Phi\left(P h^{*}\right)\right)=\operatorname{dim}\left(\Phi\left(P h_{Z}^{*}\right)\right)+\operatorname{dim}\left(\Phi\left(P h_{S}^{*}\right)\right)
$$

where "dim" means dimension. Thus we have reduced the theorem to the corresponding statement for $h_{z}$ and for $h_{s}$. Since $Z$ is a torus, $P h_{Z}^{*}=h_{Z}^{*, 1}$. Write the group operation on $Z$ additively and define $\tilde{h}_{z}(x)=h_{z}(x)-x$ for $x \in Z$, then $\Phi_{0}\left(h_{z}\right)=\operatorname{Ker}_{o}\left(\widetilde{h}_{z}\right)$. By 2.2 and 2.3, the dimension of the topological group $\operatorname{Ker}_{o}\left(\tilde{h}_{Z}\right)$ is equal to 
the dimension of the vector space $\operatorname{Ker}\left(\widetilde{h}_{Z}^{*, 1}\right)$ and therefore to the dimension of $\Phi\left(h_{Z}^{*, 1}\right)$. Since $S$ is semisimple, Aut $(S) / \operatorname{Inn}(S)$ is a finite group so 3.1 establishes the result for $h_{S}$ and completes the proof of Theorem 1.1.

The fact that the rank of $\Phi_{0}(h)$ is equal to the dimension of $P H^{*}\left(\Phi_{0}(h)\right)$ might lead one to suspect that Theorem 1.1 is a consequence of some more elaborate relationship between $H^{*}\left(\Phi_{0}(h)\right)$ and $\Phi\left(h^{*}\right)$. However, let $g \in G$ be such that the closure of the subgroup it generates is a maximal torus and let $h$ be conjugation by $g$, then $h^{*}$ is the identity isomorphism, but $\Phi_{0}(h)$ is just the maximal torus.

4. A bound on the rank. Let $\mathfrak{A}$ be a simple Lie algebra and let $\rho(\mathfrak{X})$ denote the number of algebra generators of $H^{*}(\mathfrak{N})$ which are fixed under $\eta^{*}$ for all automorphisms $\eta$ of $\mathfrak{A}$.

Proposition 4.1. Let $G$ be a compact, connected Lie group with Lie algebra \$s. Write

$$
\text { (S) } \cong 3 \oplus \mathfrak{R}_{1}^{1} \oplus \cdots \oplus \mathfrak{X}_{1}^{k(1)} \oplus \cdots \oplus \mathfrak{P}_{u}^{1} \oplus \cdots \oplus \mathfrak{X}_{u}^{k(u)}
$$

where 3 is abelian, $\mathfrak{U}_{s}^{i} \cong \mathfrak{U}_{s}^{j} \cong \mathfrak{N}_{s}$ for each $s=1,2, \cdots, u$ and all $i, j=1, \cdots, k(s)$, where $\mathfrak{X}_{s}$ is simple, and $\mathfrak{V}_{s}^{i} \not \mathfrak{N}_{t}^{j}$ if $s \neq t$. Then

$$
\sum_{s=1}^{u} \rho\left(\mathfrak{N}_{s}\right) \leqq \operatorname{rank} \Phi_{o}(h)
$$

for all automorphisms $h$ of $G$.

Proof. By Theorem 1.1, the rank of $\Phi_{0}(h)$ is equal to the dimension of $\Phi\left(P h^{*}\right)$. Let $P H^{*}(\$)$ denote the image of $P H^{*}(G)$ under the deRham isomorphism. By [3; p. 257], $P h^{*}$ can be identified with the restriction of $\eta^{*}$ to $P H^{*}(\mathbb{S})$, for an automorphism $\eta$ of $\mathbb{8}$. Consequently, it is sufficient to consider each type of simple Lie algebra in (S) by itself. That is, it is enough to prove that if $G$ is a Lie group such that $\mathbb{B} \cong \mathfrak{A} \oplus \cdots \oplus \mathfrak{A}$ ( $k$ factors) where $\mathfrak{A}$ is simple, and $h$ is an automorphism of $G$, then the rank of $\Phi_{o}(h)$ is at least $\rho(\mathfrak{A})$. In this case, the matrix $M$ of the restriction of $\eta^{*}$ to $P H^{*}(\mathbb{S})$ contains $k$ rows corresponding to each generator of $P H^{*}(\mathfrak{U})$. If the generator is one which contributes to $\rho(\mathfrak{H})$, then those same rows of $M-E$ ( $E$ the identity matrix) are linearly dependent. Thus the multiplicity of +1 as an eigenvalue of $M$ is at least $\rho(\mathfrak{A})$ and we conclude that the dimension of $\Phi\left(P h^{*}\right)$ for any automorphism $h$ of such a group $G$ is indeed at least $\rho(\mathfrak{Z})$.

If $G$ is a simply-connected compact Lie group with its Lie algebra (S) as in the proposition, then there is an automorphism $h$ of $G$ for which the rank of $\Phi_{0}(h)$ is precisely $\sum_{s=1}^{u} \rho\left(\mathscr{U}_{s}\right)$, so Proposition 4.1 
cannot be improved in general.

By [3, p. 258], which is just a restatement of pp. 81-82 of [10], we have the following table:

\begin{tabular}{l|l}
\hline type of $\mathfrak{A}$ & $\rho(\mathfrak{I})$ \\
\hline$A_{r} r$ even & $r / 2$ \\
$A_{r} r \geqq 3$ odd & $(r+1) / 2$ \\
$D_{4}$ & 2 \\
$D_{r} r \geqq 5$ & $r-1$ \\
$E_{6}$ & 4 \\
all others & $\operatorname{rank}(\mathfrak{I})$ \\
\hline
\end{tabular}

Observing that $\rho(\mathfrak{U}) \geqq 1$, we have the following known result.

CoRollary 4.2 (de Siebenthal [8]). If $G$ is a compact, connected Lie group and there is an automorphism of $G$ with a finite set of fixed points, then $G$ is abelian.

More generally, we see that knowledge of the rank of $\Phi_{o}(h)$ for an automorphism $h$ of $G$ implies restrictions on the Lie algebra of $G$ by means of the table above. For example, since $\rho(\mathfrak{i})=1$ only if $\mathfrak{Z}$ is of type $A_{1}$ or type $A_{2}$, then

COROLlARY 4.3. If there is an automorphism $h$ of a compact, connected Lie group $G$ such that $\Phi_{0}(h)$ is a sphere, then either $G$ is abelian or its Lie algebra (B) is of the form $\mathbb{B} \cong \mathbb{3} \oplus \mathfrak{X} \oplus \cdots \oplus \mathfrak{X}$ where 3 is abelian and $\mathfrak{X}$ is a simple Lie algebra, either of type $A_{1}$ or of type $A_{2}$.

5. The power map. Let $G$ be a Lie group whose components are compact. In other words, $G$ is an extension of a compact, connected Lie group $G_{0}$ by a discrete, but not necessarily finite, group. We define the rank of a component $K$ of $G$ to be the rank of the identity component of the centralizer of $g$ in $G$, for some element $g$ of $K$. To see that the definition is independent of the choice of the element, let $C_{g}: G_{0} \rightarrow G_{0}$ be conjugation by $g$ and notice that the identity component of the centralizer of $g$ is $\Phi_{o}\left(C_{g}\right)$. A path in $K$ from $g$ to any other point $g^{\prime}$ induces a homotopy from $C_{g}$ to $C_{g^{\prime}}$, so $C_{g}^{*}=C_{g^{\prime}}^{*}$. Since Theorem 1.1 states that the rank of $\Phi_{o}\left(C_{g}\right)$ can be computed from $C_{g}^{*}$, the theorem implies that the rank of a component is independent of the choice of element used to define it.

When $G$ is compact, the definition of the rank of a component 
which we have just given agrees with the definition we used in [2] because, by [7; 1.2], a maximal torus of the centralizer of $g \in K$ is the identity component of a Cartan subgroup generated from $K$.

The "power map" $p_{k}: G \rightarrow G, k \geqq 2$, is defined by $p_{k}(g)=g^{k}$. The component of $G$ containing an element $g$ is $g G_{0}$, so $p_{k}\left(g G_{0}\right) \subseteq g^{k} G_{0}$. We will establish necessary and sufficient conditions for $p_{k}\left(g G_{0}\right)=g^{k} G_{0}$.

Proposition 5.1. The degree of the map $p_{k}: g G_{0} \rightarrow g^{k} G_{0}$ is nonzero if and only if $\operatorname{rank}\left(g G_{0}\right)=\operatorname{rank}\left(g^{k} G_{0}\right)$.

Proof. Let $A=P C_{g}^{*}$, then by Theorem 1.1 the rank of $g G_{0}$ is the dimension of $\Phi(A)$. Define $A^{(k)}=A^{k-1}+A^{k-2}+\cdots+A+E$, then $A^{(k)}$ is nonsingular if and only if $\Phi(A)=\Phi\left(A^{k}\right)$. Thus rank $\left(g G_{0}\right)=$ rank $\left(g^{k} G_{0}\right)$ if and only if $A^{(k)}$ is nonsingular. By $[2 ; 2.3]$ (note the remark following that theorem), the determinant of $A^{(k)}$ is the degree of $p_{k}: g G_{0} \rightarrow g^{k} G_{0}$.

Following a suggestion of K. H. Hofmann, we define, for $g \in G$ and $k \geqq 2$, a map $\varphi_{k}^{g}$ : $G_{0} \rightarrow G_{0}$ by $\varphi_{k}^{g}(x)=g^{-k}(g x)^{k}$. Observe that the degree of $\varphi_{k}^{g}$ is equal to the degree of $p_{k}: g G_{0} \rightarrow g^{k} G_{0}$. We next wish to prove that if the degree of $\varphi_{k}^{g}$ is zero, then the dimension of $\varphi_{k}^{g}\left(G_{0}\right)$ is less than the dimension of $G_{0}$. We establish the usual special cases first.

LEMMA 5.2. Let G be a Lie group in which the identity component $G_{0}$ is a torus. If the degree of $\varphi_{k}^{g}$ is zero, then $\operatorname{dim}\left(\varphi_{k}^{g}\left(G_{0}\right)\right)<\operatorname{dim}\left(G_{0}\right)$.

Proof. Abbreviate $\varphi_{k}^{g}$ as $\varphi$ and let $G_{0}^{\wedge}$ be the character group of $G_{0}$. Just as we did in the proof of Proposition 2.3, we may identify $\varphi^{\wedge}$ with $\varphi_{J}^{*}: H^{1}\left(G_{0} ; J\right) \rightarrow H^{1}\left(G_{0} ; J\right)$. Again let $C_{g}$ denote conjugation by $g$ and set $A=P C_{g}^{*}$. Noting that

$$
\varphi(x)=C_{g}^{k-1}(x) \cdot C_{g}^{k-2}(x) \cdots C_{g}(x) \cdot x,
$$

an induction argument shows that $\varphi_{j}^{*}$ may be identified with $A^{(k)}=$ $A^{k-1}+A^{k-2}+\cdots+A+E$ so the hypothesis implies that $\varphi^{\wedge}$ has a nontrivial kernel. We have an exact sequence

$$
0 \longrightarrow \operatorname{Ann}\left(\varphi\left(G_{0}\right)\right) \longrightarrow G_{0}^{\wedge} \stackrel{i^{\wedge}}{\longrightarrow} \varphi\left(G_{0}\right)^{\wedge} \longrightarrow 0
$$

where Ann $\left(\varphi\left(G_{0}\right)\right) \neq 0$ since it contains the kernel of $\varphi^{\wedge}$. Thus $\varphi\left(G_{0}\right)$ is a proper subtorus of $G_{0}$ and consequently it is of lower dimension.

Let $G$ be a Lie group with compact components and suppose $g \in G$ has the property that $g^{m}$ is in the centralizer of $G_{0}$; for some $m \geqq 1$. Let $\Gamma=\mathrm{U}_{r=0}^{m-1} g^{r} G_{0}$ and define operation "o" on $\Gamma$ by

$$
\left(g^{s} x\right) \circ\left(g^{t} y\right)=g^{\psi(s+t)} z
$$


where $\left(g^{s} x\right)\left(g^{t} y\right)=g^{s+t} z$ in $G\left(x, y, z \in G_{0}\right)$ and $\psi$ is reduction modulo $m$. Consider the subgroup $G^{g}=\bigcup_{r} g^{r} G_{0}$ of $G$ and define $\Psi: G^{g} \rightarrow \Gamma$ by $\Psi\left(g^{s} x\right)=g^{\psi(s)} x$. Since we can compute that

$$
\Psi\left(\left(g^{s} x\right)\left(g^{t} y\right)\right)=\Psi\left(g^{s} x\right) \circ \Psi\left(g^{t} y\right),
$$

we conclude that $\Gamma$ is a group and $\Psi$ is a homomorphism.

LeMma 5.3. Let $G$ be a Lie group in which $G_{0}$ is compact and semisimple. If the degree of $\varphi_{k}^{g}$ is zero, then $\operatorname{dim}\left(\varphi_{k}^{g}\left(G_{0}\right)\right)<\operatorname{dim}\left(G_{0}\right)$.

Proof. Since $G_{0}$ is semisimple, we may assume that $g^{m}$ is in the centralizer of $G_{0}$, for some $m \geqq 1$. The computation above proves the commutativity of the diagram

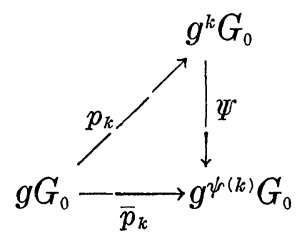

where $\bar{p}_{k}$ denotes the power map in $\Gamma$. The hypothesis thus implies that the degree of $\bar{p}_{k}$ is zero so, since $\Gamma$ is a compact Lie group, the proof of Theorem 5.2 of [2] establishes that $\operatorname{dim}\left(\bar{p}_{k}\left(g G_{0}\right)\right)<\operatorname{dim}\left(G_{0}\right)$. Observing that $p_{k}\left(g G_{0}\right)=g^{k} \Phi_{k}^{g}\left(G_{0}\right)$ completes the argument.

Proposition 5.4. Let $G$ be a Lie group with compact components. If the degree of $\varphi_{k}^{g}$ is zero, then $\operatorname{dim}\left(\varphi_{k}^{g}\left(G_{0}\right)\right)<\operatorname{dim}\left(G_{0}\right)$.

Proof. Returning to the notation of $\S 3$, we write $G_{0}=Z S$ where $Z$ is abelian and $S$ is semisimple, and for $\varphi=\varphi_{k}^{g}$, we let $\varphi_{Z}$ and $\varphi_{S}$ be the restrictions (note that $\phi$ is a product of automorphisms). Since $P \varphi^{*}=P \varphi_{Z}^{*} \oplus P \varphi_{S}^{*}$, the degree of $\varphi$ is the product of the degrees of $\varphi_{Z}$ and $\varphi_{S}$; so at least one must vanish by the hypothesis. Thus by 5.2 applied to the subgroup of $G$ generated by $g$ and $Z$, if the degree of $\varphi_{Z}$ is zero then $\operatorname{dim}\left(\varphi_{Z}(Z)\right)<\operatorname{dim}(Z)$. Similarly, if the degree of $\varphi_{S}$ is zero, then $\operatorname{dim}\left(\varphi_{S}(S)\right)<\operatorname{dim}(S)$ by 5.3. Let $\mu: Z \times S \rightarrow G_{0}$ be defined by $\mu(z, s)=z s$, then since $Z$ is central, $\phi \mu=\mu\left(\varphi_{Z} \times \varphi_{S}\right)$ and we have

$$
\operatorname{dim}\left(\varphi\left(G_{0}\right)\right)=\operatorname{dim}(\varphi(Z))+\operatorname{dim}(\varphi(S))<\operatorname{dim}\left(G_{0}\right) .
$$

Proposition 5.1 and 5.4 together imply the following extension of the main result, Theorem 5.2, of [2].

THEOREM 5.5. Let $G$ be a Lie group with compact components. 
The statements below are equivalent:

(i ) $p_{k}\left(g G_{0}\right)=g^{k} G_{0}$.

(ii) The degree of $p_{k}: g G_{0} \rightarrow g^{k} G_{0}$ is not zero.

(iii) $\operatorname{rank}\left(g G_{0}\right)=\operatorname{rank}\left(g^{k} G_{0}\right)$.

\section{REFERENCES}

1. R. Brown, Fixed points of endomorphisms of compact groups, Bull. Amer. Math. Soc., 80 (1974), 293-96.

2. - On the power map in compact groups, Quart. J. Math., 22 (1971), 395-400.

3. - The real cohomology of compact disconnected Lie groups, Proc. Amer. Math. Soc., 37 (1973), 255-59.

4. H. Hopf. Über den Rang geschlossener Liescher Gruppen, Comment. Math. Helv., 13 (1940), 119-43.

5. —, Über die Topologie der Gruppen-Mannigfaltigkeiten und ihre Verallgemeinerung, Ann. Math., 42 (1941), 22-52.

6. L. Pontryagin, Topological Groups, second edition, Gordon and Breach, 1966,

7. G. Segal, The representation-ring of a compact Lie group, Publ. Math. I. H. E. S., 34 (1968), 113-28.

8. J. de Siebenthal, Sur les groupes de Lie compactes non connexes, Comment. Math. Helv., 31 (1956), 41-89.

9. N. Steenrod, Universal homology groups, Amer. J. Math., 58 (1936), 661-701.

10. R. Steinberg, Endomorphisms of linear algebraic groups, Mem. Amer. Math. Soc., 80 (1968).

Received April 30, 1975. This research was partially supported by NSF Grant MPS 75-04914.

University of California, Los ANgeles, California 



\section{PACIFIC JOURNAL OF MATHEMATICS}

EDITORS

RICHARD ARENS (Managing Editor)

University of California

Los Angeles, California 90024

R. A. BEAUMONT

University of Washington

Seattle, Washington 98105
J. DugundJI

Department of Mathematics

University of Southern California

Los Angeles, California 90007

D. Gilbarg and J. Milgram

Stanford University

Stanford, California 94305

\section{ASSOCIATE EDITORS}

E. F. BECKENBACH

B. H. NeumanN

F. WOLF

K. YosHIDA

\section{SUPPORTING INSTITUTIONS}

UNIVERSITY OF BRITISH COLUMBIA

UNIVERSITY OF SOUTHERN CALIFORNIA

CALIFORNIA INSTITUTE OF TECHNOLOGY

UNIVERSITY OF CALIFORNIA

STANFORD UNIVERSITY

UNIVERSITY OF TOKYO

MONTANA STATE UNIVERSITY

UNIVERSITY OF UTAH

UNIVERSITY OF NEVADA

WASHINGTON STATE UNIVERSITY

NEW MEXICO STATE UNIVERSITY

UNIVERSITY OF WASHINGTON

OREGON STATE UNIVERSITY

UNIVERSITY OF OREGON

OSAKA UNIVERSITY

AMERICAN MATHEMATICAL SOCIETY
NAVAL WEAPONS CENTER

Printed in Japan by International Academic Printing Co., Ltd., Tokyo, Japan 


\section{Pacific Journal of Mathematics}

\section{Vol. 63, No. $1 \quad$ March, 1976}

Ralph Artino, Gevrey classes and hypoelliptic boundary value problems ....... 1

B. Aupetit, Caractérisation spectrale des algèbres de Banach commutatives .... 23

Leon Bernstein, Fundamental units and cycles in the period of real quadratic

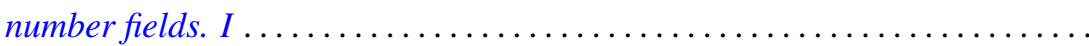

Leon Bernstein, Fundamental units and cycles in the period of real quadratic number fields. II.................................... 63

Robert F. Brown, Fixed points of automorphisms of compact Lie groups ........

Thomas Ashland Chapman, Concordances of noncompact Hilbert cube

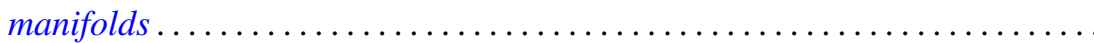

William C. Connett, V and Alan Schwartz, Weak type multipliers for Hankel

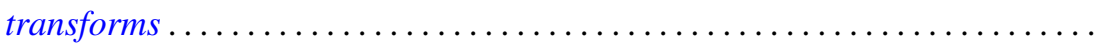

John Wayne Davenport, Multipliers on a Banach algebra with a bounded approximate identity .....................................

Gustave Adam Efroymson, Substitution in Nash functions ................ 137

John Sollion Hsia, Representations by spinor genera ..................

William George Kitto and Daniel Eliot Wulbert, Korovkin approximations in

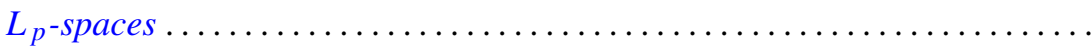

Eric P. Kronstadt, Interpolating sequences for functions satisfying a Lipschitz. condition ...........................................

Gary Douglas Jones and Samuel Murray Rankin, III, Oscillation properties of certain self-adjoint differential equations of the fourth order...

Takaŝi Kusano and Hiroshi Onose, Nonoscillation theorems for differential

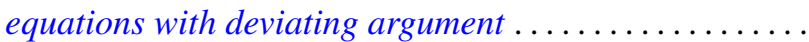

David C. Lantz, Preservation of local properties and chain conditions in commutative group rings. ...

Charles W. Neville, Banach spaces with a restricted Hahn-Banach extension property....

Norman Oler, Spaces of discrete subsets of a locally compact group ...

Robert Olin, Functional relationships between a subnormal operator and its minimal normal extension.

Thomas Thornton Read, Bounds and quantitative comparison theorems for nonoscillatory second order differential equations ...... .

Robert Horace Redfield, Archimedean and basic elements in completely distributive lattice-ordered groups...

Jeffery William Sanders, Weighted Sidon sets

Aaron R. Todd, Continuous linear images of pseudo-complete linear topological spaces.

J. Jerry Uhl, Jr., Norm attaining operators on $L^{1}[0,1]$ and the Radon-Nikodým property.

William Jennings Wickless, Abelian groups in which every endomorphism is a left multiplication. 